\title{
Comparison of gut microbiome from Sulawesi carrion and burying beetles (Coleoptera: Silphidae) through metagenomic analysis of $16 S$ rRNA gene
}

\author{
Ruby Setiawan ${ }^{1, *}$, I Nyoman Sumerta ${ }^{1}$, Syahfitri Anita $^{2}$, Raden Pramesa Narakusumo ${ }^{2}$, and Anang Setiawan Achmadi ${ }^{2}$ \\ ${ }^{1}$ Indonesia Culture Collection, Microbiology Division, Research Center for Biology, Indoesia Insititute of Sciences (LIPI), Jalan Raya \\ Jakarta Bogor Km. 46, Cibinong, Indonesia 16911 \\ ${ }^{2}$ Museum Zoologicum Bogoriense, Zoology Division, Research Center for Biology, Indonesian Institute of Sciences (LIPI), Jalan Raya \\ Bogor Km. 46, Cibinong, Indonesia 16911
}

\begin{abstract}
Carrion beetle (Coleoptera: Silphidae) is one of the scavengers which obtain nutrition from carcass decomposition which supported by the microbial symbionts through the mutual symbiosis. In this study, we characterized and compared the gut microbial community from the species of Nicrophorinae (Nicrophorus distinctus Grouvelle, 1885) and Silphinae (Necrophila renatae Portevin, 1920) from Dako Mountain, Central Sulawesi using 16S metagenomic approach. A total of 661 bacterial Operational Taxonomic Units (OTUs) at the species level were obtained from the guts of Ni. distinctus and Ne. renatae. Those numbers were predominated by Firmicutes and Proteobacteria, followed by Bacteriodetes in both species. Interestingly, a high number of Lactobacillales was observed in Ni. distinctus and lower number in Clostridiales and Cardiobacteriales compared to Ne. renatae, which showed higher abundance of those classes. Both of these insect species have nearly the same microbial diversity values, even though there some lower taxa levels were found different abundance. These results suggest that the patterns of the gut microbial structure depicted their roles in certain behavior and habitat on decomposing carcasses and could be correlated to the specific level of taxa roles in nutrient processing.
\end{abstract}

\section{Introduction}

Beetles from family Silphidae is one of the most important scavengers in the nutrient cycling on terrestrial environment. They provide ecosystem services by recycling the nutrients from decomposing carcasses. Silphidae family consists of two subfamilies which are Silphinae (carrion beetles) and Nicrophorinae (burying beetles). Those groups have different behaviour on treating carcass. The carrion beetles feed and rear their offspring inside the carcass while the burying beetles rear their offspring around the buried carcass to hide it from other scavengers [1]. In decomposing process, they are also supported by microbes to digest the substrates.

The carrion and burying beetles possess inter-specific relations with microorganism. The population of microbes inside their beetle body might play important role to control metabolism process of decaying carcass. For example, microorganisms could use carrion as a source of carbohydrates, lipids and proteins as toxin production to reduce carrion quality so that carcasses can be easily digested. This immune system and antimicrobial characteristics provided by microorganism could help them to survive in particular habitat and the immune system of Silphidae beetles particularly from Nicrophorus vespilloides have been well studied [2-9]. Therefore, this study was essential to reveal the microbial community structure that illustrating their microbial support in decaying carcass in their body.

The microbial community in insect gut has crucial roles on their digestion process. Gut microbiome drives multiple aspects of an insect's ecology, such as nutrients acquisition, antimicrobial production, and the protection of the gut from invading pathogens, and systemic changes in immune capacity [10-14]. For instance, insects could have assistance from their antibioticproducing bacteria to defend their body from pathogen fungus $[15,16]$. However, the structure of insect gut microbiome depends on their habitat, environment condition, and diet $[17,18]$ which can support their life in different ways. Nowadays, study in gut microbiome receives wide attention. It is because the insectassociated microbiome could result in practical applications, such as insect pest management, insect vector to control the carried pathogens, waste water treatments, prebiotic, and beneficial insects conservation

\footnotetext{
* Corresponding author: rubysbio@gmail.com
} 
[19]. Also, it could be applied for health and drug development [20].
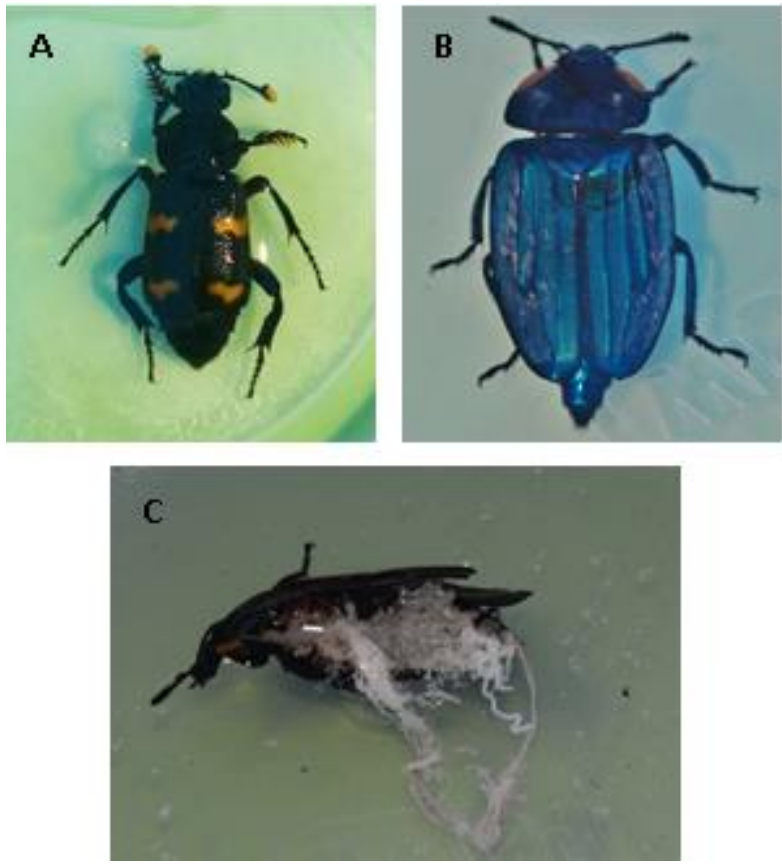

Fig. 1. Nicrophorus distinctus (A), Necrophila renatae (B) and Ni.distinctus after dissected (C)

Study gut microbiome of carrion and burying beetles with advance technology such as $16 \mathrm{~S}$ metagenomic could be beneficial for completing the previous research. This study aimed at comparing the microbial community structure of two subfamilies of Shilpidae which has different behaviour on treating carcass which are Nicrophorus distinctus Grouvelle, 1885 and Necrophila renatae Portevin, 1920. Those species samples were collected form Dako Mountain, Sulawesi. By using metagenomic analysis, we expect that the whole community could be explained and compared. Then, we could obtain the comprehensive insight about interrelationship of each species in decaying carcass.

\section{Material and methods}

\subsection{Sample collection and preparation}

We collected adults stage of carrion and burying beetles using carrion-baited pitfall traps in Dako Mountain, ToliToli regency, Central Sulawesi Province, Indonesia (N 1³'46.89"; E 12053'56.01"). Samples were preserved in $70 \%$ ethanol and two beetles were identified as $\mathrm{Ni}$. distinctus and $\mathrm{Ne}$. renatae. As for bacterial DNA profiling, we dissected beetle specimens by rinsing prior in sterile water to remove surface contaminants and then sterilized in absolute ethanol for several times. Two representative samples of those species were dissected to collect the whole gut and then stored in absolute ethanol for further analysis.

\subsection{Bacterial community profiling}

The two bacterial DNA samples from guts of $\mathrm{Ni}$. distinctus and Ne. renatae were extracted using ZymoBIOMICS DNA Miniprep Kit according to manufacturer's instructions. About $5 \mathrm{ng}$ of extracted DNA were subjected to PCR to amplify V3-V4 region of $16 \mathrm{~S}$ rDNA using primer V3-f (5'CTACGGGNGGCWGCAG-3') and V4-r (5'GACTACHVGGGTATCTAATCC-3') [21]. Amplification were carried out using MyTaq HS DNA Polymerase (BIO-25049, Bioline, UK). The PCR products were sent to sequencing service provider (Macrogen, Korea) for indexing and library preparation were conducted according to Illumina 16S Metagenomic Sequencing Library Preparation.

The obtained sequences were analyzed using QIIME2-2019.4 pipeline [22]. Denoising and dereplicating were carried out using DADA2 plugin [23]. All amplicon sequence variants (ASVs) were filtered using q2-feature-table plugin and the ASVs were less than 10 sequence reads omitted. Furthermore, the q2-phylogeny plugin was used to align the filtered ASVs using mafft [24] and construct phylogeny using Fasttree 2 [25]. As a result, phylogenetic tree reconstruction was carried out from 20 most abundant OTU from each Silphidae species and 10 most shared OTU and analyzed according to Kaltenpoth and Steiger (2013) followed by minimal ancestor deviation (MAD) method (Tria et al. 2017) and visualized using iTOL v4 (Letunic and Bork 2019) [26]. Subsequently, the taxonomy profiles were assigned using q2-feature-classify classify-sklearn naive Bayes taxonomy [27] against SILVA 132 reference sequences at $99 \%$ similarity. Meanwhile, the diversity values were calculated using vegan package [28] and shared OTUs through Venn diagram using VennDiagram package [29].

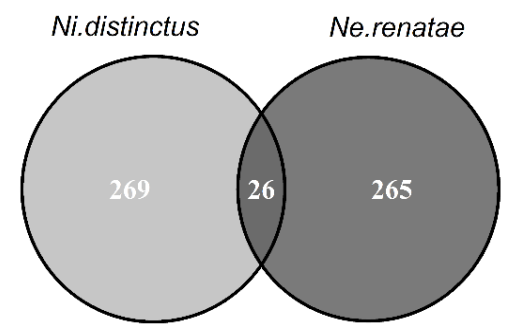

Fig. 2. Number of OTUs and shared OTUs from Silphidae gut microbiome

\section{Results and discussion}

About 260,611 sequences were obtained after metagenomic sequencing of bacterial 16S rRNA gene. After quality filtering, trimming, merging and chimera removal using DADA2 pipeline in QIIME2, a total of 146,112 sequences binned into 661 OTUs, 101 OTUs were omitted because the abundance was below the threshold of 10 sequence reads per OTU. The total 661 OTUs classified into 4 Phyla, 9 Class, 17 Order, 23 Family, 38 Genus, 56 Species. Overall, the diversity 

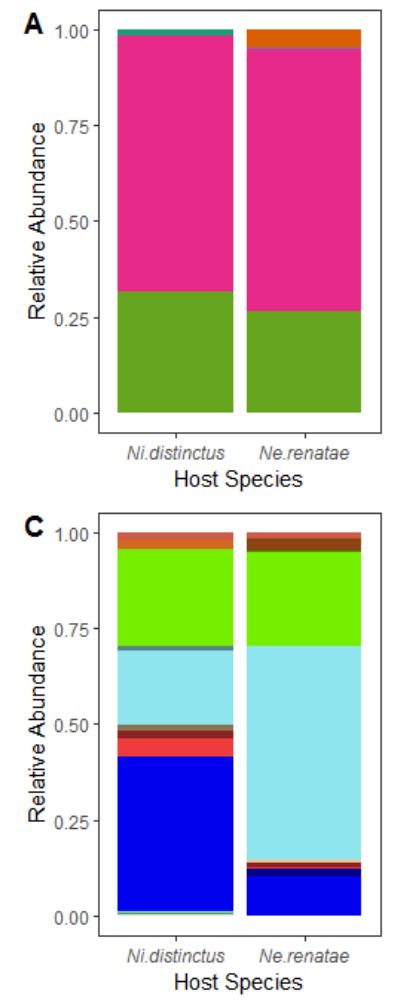
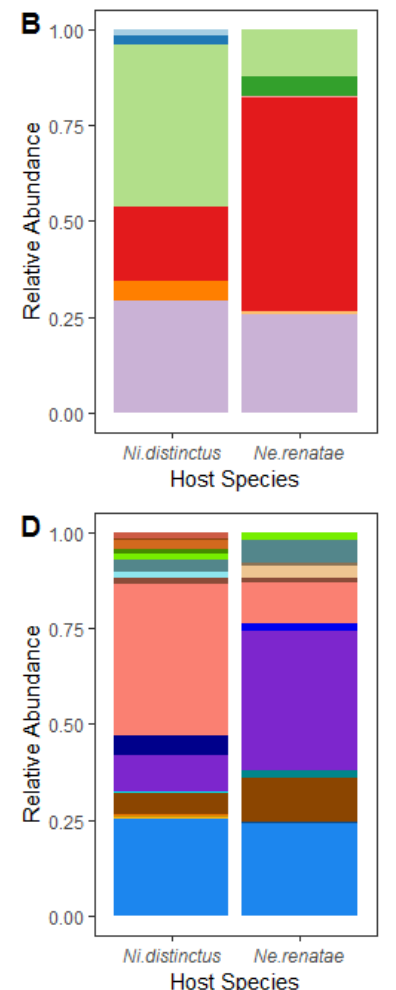

Host Species
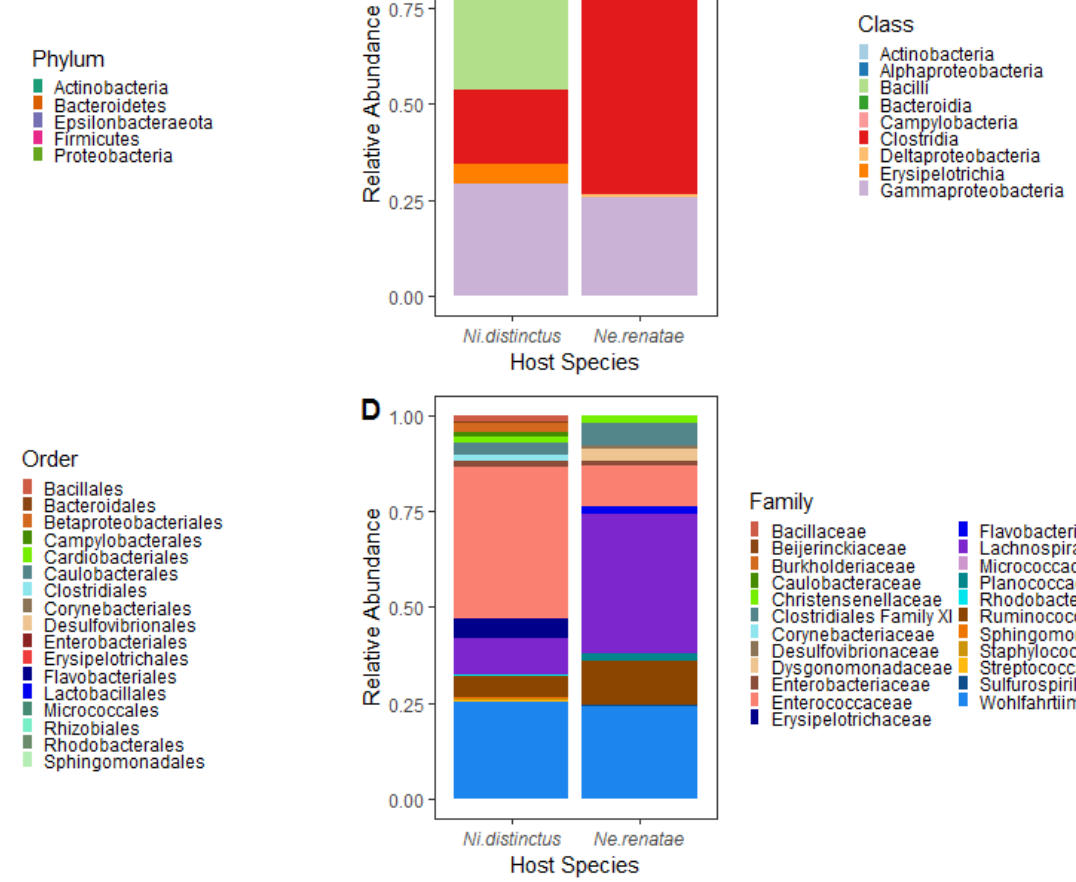

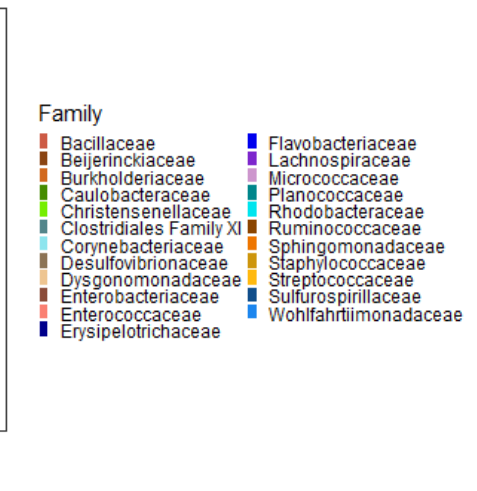

Fig. 3. Relative abundance of Silphidae gut microbiome in Phylum level (A), Class level (B), Order level (C) and Family level (D)

value of the Shannon index from Ni. distinctus and Ne. renatae were 3.81 and 3.96 whereas the Pielou's Evenness values were 0.671 and 0.697 , respectively. This result shows the diversity and the abundance of both samples were nearly the same. Shukla et al (2018) also reported the number of Shannon index and evenness value from $\mathrm{Ni}$. Vespilloides were 3.7 and 0.6 , respectively [30]. This diversity value could be as adaptation of these species with their decomposing activities since they come from the same family. On the other hand, the number of shared OTUs from each sample were very low, about $3.9 \%$ from total OTUs (Figure 1), showing these insects have different gut microbiome composition with almost the same number of OTUs 269 and 265 for Ni. distinctus and Ne. renatae, respectively.

The gut microbiome from $\mathrm{Ni}$. distinctus and $\mathrm{Ne}$. renatae was predominated by Firmicutes about $66 \%$ and $68 \%$ and Proteobacteria about $31 \%$ and $26 \%$ (Figure 3 ). The separation was seen from the Class level, the Bacilli was predominantly in $\mathrm{Ni}$. distinctus around $41 \%$ while Clostridia was in Ne. renatae almost $56 \%$. In $\mathrm{Ni}$. distinctus, Order of Lactobacillales mainly consists of Enterococcaceae about 39\% whereas Lachnospiraceae rich in Ne. renatae gut around 36\%. Degenkolb, Düring, and Vilcinskas (2011) explained that the high abundance of Lactobacillales in $\mathrm{Ni}$. distinctus might play a role in the carcass preservation since its behavior is tend to monopolize the carcass. Wohlfahrtiimonadaceae was found in both Ni. distinctus and Ne. Renatae as the second largest family of gut microbiome about $25 \%$ and $24 \%$, respectively [31].

The composition of gut microbiome of $\mathrm{Ni}$. distinctus and $N e$. renatae shows that the differences between those species which refer to the behavior of carrion and burying beetles. We assumed that their way rears carcass is related to their differences of gut microbial community structure. Since each species has its certain microbial community pattern, it might release different substances treating the carcass. For instance, gut microbiome of Silphidae species could nurture their offspring by maintaining the putrefaction rate of carcass [30]. Their gut microbiome provides nutrient and modify the natural decaying microbes by secreting any substances for larva development whether they are nurtured inside or outside of the carcass.

Although the predominant phyla were in the same groups, their lower taxa levels were vary in abundance and taxa composition. The composition of Ni. distinctus and Ne. Renatae genera can be seen on the phylogenetic tree (Figure 4). Their guts consisted of Vagococcus, Bacillus, Tissierella, Sporanarobacter, Clostridium, Corynebacterium, Brevundimonas, Sutterella, Cedecea, Wohlfahrtiimonas, Ignatzschineria, Myroides and Anaerorhabdus. Furthermore, Vagococcus was the most abundant genera in Ni. distinctus whereas Clostridium was predominant in Ne. renatae. Some genera might be not cultured yet and by this advance technology, we can view in deeper way to explore those uncultured species for further study.

The comprehensive number of genera that explained by $16 \mathrm{~S}$ metagenomic analysis are able to create a holistic view about their roles from several previous studies. Kaltenpoth and Steiger (2013) studied about the microbial communities of Silphidae in hindgut [18]. They reported that high abundance of Enterococcaceae and Clostridiales in the Nicrophorus and Necrophila hindgut, respectively. The Enterococcaceae and 


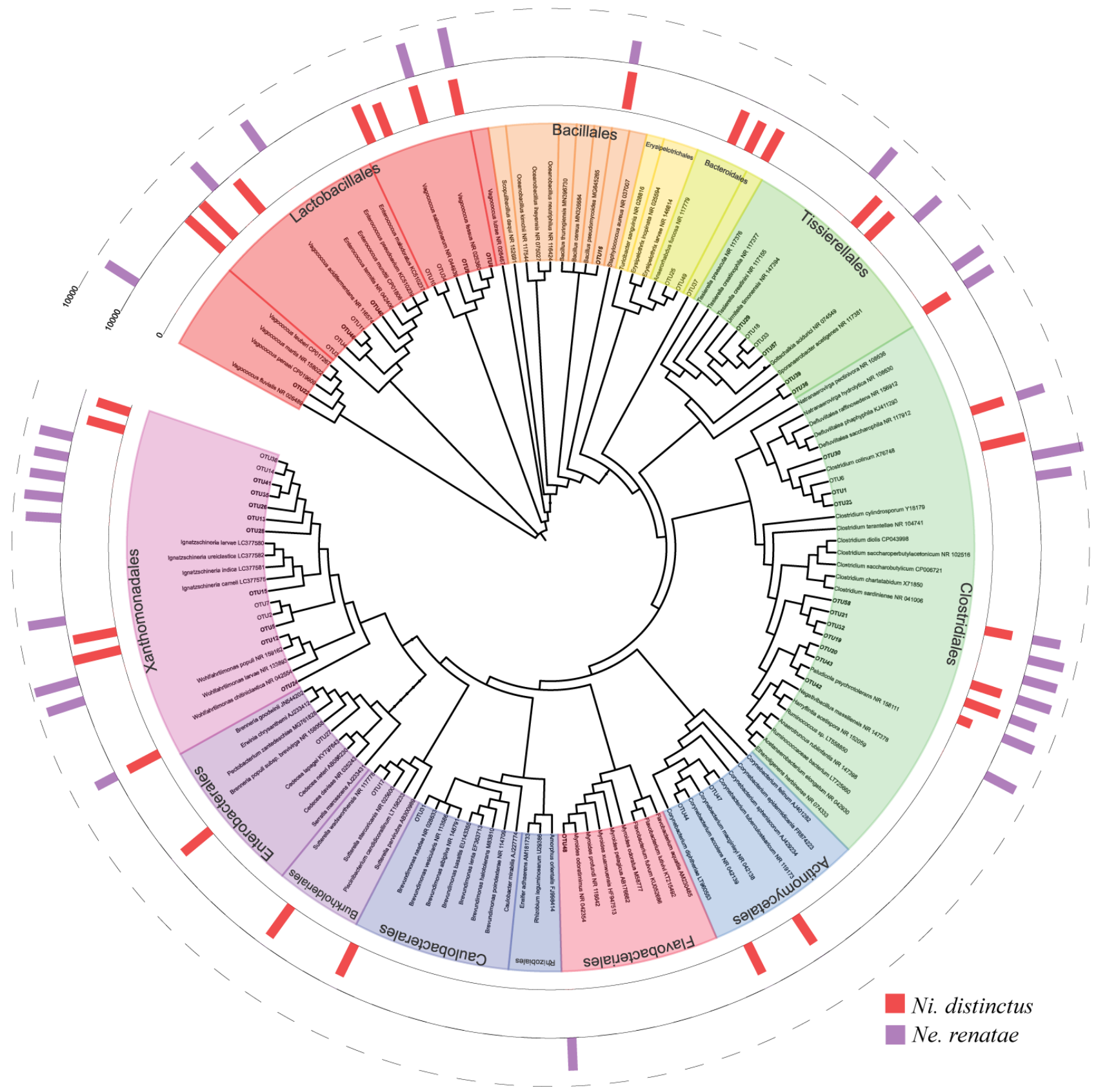

Fig. 4. Phylogenetic tree of the selected OTU from Silphidae. The bar chart outside the tree shows the abundance of the representative OTUs

Clostridiales group possses roles while some species of Clostridiales such as Tissierella can degrade the creatine, and provide nutrition for the host [32]. Meanwhile, the Enterococcaceae that was highly abundant in $\mathrm{Ni}$. distinctus and also found in Ne. renatae were known to produce bacteriocins or other antimicrobials [33]. The genera of Ignatzschineria and Wohlfahrtiimonas from the Xanthomonadaceae have several enzymatic activities such as urease, esterase and lipase that could promote the carcass digestion [34-36]. When the creatine of carcass in carcass could not degraded by insect, the insects could utilize the bacterial enzymes for degradation process.

\section{Conclusion}

This is the first study using an advance method to delineate the microbial community in carrion and burying beetles (Coleoptera: Silphidae) which result in unique patterns of microbial community structures instead of almost the same diversity values. These structures could be as patterns from their particular behavior and roles in decomposing carcass leading to different abundance in lower taxa level, such as Lactobacillales, Clostridiales, and Cardiobacteriales. 
We would like to express our gratitude to Mrs. Maya Komalasari with her great laboratory work on extracting the DNA of gut bacteria and this study was funded by the DIPA of PDII-LIPI fiscal years 2018.

\section{References}

1. M.P. Scott, Annu Rev Entomol, 43, 595-618 (1998) https://doi.org/10.1146/annurev.ento.43.1.595

2. D.E. Rozen, D.J.P. Engelmoer, P.T. Smiseth,. Proc Natl Acad Sci USA, 105, 17890-17895. (2008) https://doi.org/10.1073/pnas.0805403105

3. S.C. Cotter, R.M. Kilner, Behav Ecol, 21, 663-668 (2010) https://doi.org/10.1093/beheco/arq070

4. S.C. Cotter, R.M. Kilner, J Anim Ecol, 79, 35-43 (2010) 2656.2009.01593.x

https://doi.org/10.1111/j.1365-

5. 5. S.C. Cotter, E. Topham, A.J.P. Price, R.M. Kilner,.Ecol Lett, 13, 1114-1123 (2010). https://doi.org/10.1111/j.1461-0248.2010.01500.x

6. S.C. Cotter, J.E. Littlefair, P.J. Grantham, R.M. Kilner, J Anim Ecol, 82, 846-853 (2013) https://doi.org/10.1111/1365-2656.12047

7. C.L. Hall, N.K. Wadsworth, D.R. Howard, E.M. Jennings, L.D. Farrell, T.S. Magnuson, R.J. Smith, Environmental Entomology, 40, 669-678 (2011) https://doi.org/10.1603/EN10137

8. C.E. Reavey, N.D. Warnock, H. Vogel, S.C. Cotter, Behav Ecol, 25, 415-423. (2014) https://doi.org/10.1093/beheco/art127

9. C.E. Reavey, N.D. Warnock, A.P. Garbett, S.C. Ecol Evol, 5, 4365-4375 https://dx.doi.org/10.1002\%2Fece3.1668

10. M. Kaltenpoth, Trends Microbiol, 17, 529-535 (2009) https://doi.org/10.1016/j.tim.2009.09.006

11.D.R. Colman, E.C. Toolson, C.D. Takacs-Vesbach, Mol Ecol, 21, 5124-5137 (2012) https://doi.org/10.1111/j.1365-294X.2012.05752.X

12. R. Seipke, J. Barke, M.X. Ruiz-Gonzalez, J. Orivel, D.W. Yu, M.I. Hutchings, Antonie van Leeuwenhoek, 101, 443-447 (2012) https://doi.org/10.1007/s10482-011-9621-y

13.P. Engel, N.A. Moran, FEMS Microbiol Rev, 37, 699-735 (2013) $\quad$ https://doi.org/10.1111/1574$\underline{6976.12025}$

14.M.W. Shao, Y.H. Lu, S. Miao, Y. Zhang, T.T. Chen, Y.L. Zhang, PloS One, 10, e0134542, (2015) https://doi.org/10.1371/journal.pone.0134542

15. A.E. Little, T. Murakami, U.G. Mueller, C.R. Currie, Biol Lett, 2, 12-16 (2006) https://doi.org/10.1098/rsbl.2005.0371

16. Klassen, J. P. (2014). Microbial secondary metabolites and their impacts on insect symbioses. Current Opinion in Insect Science. 6. http://dx.doi.org/10.1016/j.cois.2014.08.00.

17.D. Bertino-Grimaldi, M.N. Medeiros, R.P. Vieira, $\begin{array}{llll}\text { SpringerPlus, } & \text { 2, } & 609 & \text { (2013). }\end{array}$ https://doi.org/10.1186/2193-1801-2-609

18. M. Kaltenpoth, S. Steiger, Mol Ecol, 23, 1251-1267. (2013) https://doi.org/10.1111/mec.12469

19. E. Crotti, A. Balloi, C. Hamdi, L. Sansonno, M. Marzorati, E. Gonella, G. Favia, A. Cherif, C. Bandi,
A. Alma, D. Daffonchio, 5, 307-317 (2014) https://doi.org/10.1111/j.1751-7915.2011.00312.x

20.J.M. Kinross, A.W. Darzi, J.K. Nicholson, Genome Med, 3, 14 (2011) https://doi.org/10.1186/gm228

21.A. Klindworth, E. Pruesse, T. Schweer, J. Peplies, C. Quast, M. Horn, F.O. Glöckner. Nucleic Acids Res, 41, (2013) https://doi.org/10.1093/nar/gks808

22.E, Bolyen, J.R. Rideout, M.R. Dillon, N.A. . Bokulich, C.C. Abnet, G.A. Al-Ghalith, H. Alexander, Nat Biotechnol, 37, 852-57 (2019) https://doi.org/10.1038/s41587-019-0252-6

23. B.J. Callahan, P.J. McMurdie, M.J. Rosen, A.W. Han, A.J.A Johnson, S.P. Holmes, Nat Methods, 13, 581-83 (2016) https://doi.org/10.1038/nmeth.3869

24. K. Katoh, D.M. Standley, Mol Biol Evol, 30, 772-80. (2013) https://doi.org/10.1093/molbev/mst010

25. M.G. Price, P.S. Dehal, A.P. Arkin, PloS One, 5, e9490

(2010) https://doi.org/10.1371/journal.pone.0009490

26. L. Letunic, P. Bork, Nucleic Acids Res, 47, W256W259 (2019) https://doi.org/10.1093/nar/gkz239

27.N.A. Bokulich, B.D. Kaehler, J.R. Rideout, M. Dillon, E. Bolyen, R. Knight, G.A. Huttley, JG. Caporaso, Microbiome, 6, $90 \quad$ (2018). https://doi.org/10.1186/s40168-018-0470-z.

28. J. Oksanen, F.G. Blanchet, M. Blanchet, R. Blanchet, P. Legendre, D. Legendre, P.R. Minchin, Vegan: Community Ecology Package, (2019). https://CRAN.R-project.org/package=vegan

29. H. Chen, P.C. Boutros, BMC Bioinformatics, 12, (2011). https://doi.org/10.1186/1471-2105-12-35.

30.S.P. Shukla, C. Plata, M. Reichelt, S. Steiger, D.G. Heckel, M. Kaltenpoth, A. Vilcinskas, H. Vogel, Proc Natl Acad Sci USA, 115, 11274-79 (2018) https://doi.org/10.1073/pnas.1812808115

31.T. Degenkolb, R.A. Düring, A. Vilcinskas, J Chem Ecol, 37, 724-735 https://doi.org/10.1007/s10886-011-9978-4

32. C. Harms, A. Schleicher, M.D. Collins, J.R. Andreesen, 48, 983-93 (1998). https://doi.org/10.1099/00207713-48-3-983.

33.R.B. Schmid, R.M. Lehman, V.S. Brözel, J.G. Lundgren, The Florida Entomologist, 97, 575-84 (2014). https://doi.org/10.1653/024.097.0232

34.H. Vogel, S.P. Shukla, T. Engl, B. Weiss, R. Fischer, S. Steiger, D.G. Heckel, M. Kaltenpoth, A. Vilcinskas, Nat Commun, 8, $15186 \quad$ (2017) https://doi.org/10.1038/ncomms 15186

35.E.M. Toth, P. Schumann, A.K. Borsodi, Z. Keki, A.L. Kovacs, K. Marialigeti, Int J Syst Evol Microbiol, 58, 976-981 (2008) https://doi.org/10.1099/ijs.0.65324-0.

36. J.K. Lee, Y.Y. Lee, K.H. Park, J. Sim, Y. Choi, S.J. Lee, Antonie van Leeuwenhoek, 105, 15-21. (2014) https://doi.org/10.1007/s10482-013-0048-5 